This document is the accepted manuscript version of the following article:

Fu, Y. H., Piao, S., Ciais, P., Huang, M., Menze1, A., Peaucel1e, M., ...

Janssens, I. A. (2016). Long-term linear trends mask phenological shifts.

International Journal of Biometeorology, $60(11), 1611-1613$.

https://doi .org/10.1007/s00484-016-1253-5

\title{
Long-term linear trends mask phenological shifts
}

Yongshuo H Fu123

Shilong Piao1245

Philippe Ciais6

Mengtian Huang2

Annette Menzel78

Marc Peaucelle6

Shushi Peng26

Yang Song9

Yann Vitasse 101112

Zhenzhong Zeng2

Hongfang Zhao2

Guiyun Zhou13

Josep Peñuelas 1415

Ivan A Janssens3

1.Department of EcologyPeking UniversityBeijingChina

2.Sino-French Institute for Earth System Science, College of Urban and Environmental SciencesPeking UniversityBeijingChina

3.Centre of Excellence PLECO (Plant and Vegetation Ecology), Department of BiologyUniversity of AntwerpWilrijkBelgium

4.Key Laboratory of Alpine Ecology and Biodiversity, Institute of Tibetan Plateau ResearchChinese Academy of SciencesBeijingChina

5.Center for Excellence in Tibetan Earth ScienceChinese Academy of SciencesBeijingChina

6.Laboratoire des Sciences du Climat et de l'EnvironnementCEA CNRS UVSQGifsur-YvetteFrance

7.EcoclimatologyTechnische Universität MünchenFreisingGermany

8.Institute for Advanced StudyTechnische Universität MünchenGarchingGermany

9.Department of Atmospheric SciencesUniversity of IllinoisUrbanaUSA

10.Institute of GeographyUniversity of NeuchatelNeuchatelSwitzerland

11.WSL Swiss Federal Institute for Forest, Snow and Landscape ResearchNeuchatelSwitzerland

12.WSL Institute for Snow and Avalanche Research SLF, Group Mountain EcosystemsDavosSwitzerland

13.School of Resources and EnvironmentUniversity of Electronic Science and Technology of ChinaChengduChina

14.CREAFBarcelonaSpain

15.CSIC, Global Ecology Unit CREAF-CSIC-UABBarcelonaSpain

Climate warming has substantially advanced the timing of leaf unfolding, while the temperature sensitivity of leaf unfolding $\left(\mathrm{S}_{\mathrm{T}}\right)$ was significantly reduced over the past 
three decades according to our recent study (Fu et al 2015). We are very happy to see that the article from Wang et al (2016) confirmed these finding using a 15-year window, despite using only 927 species-sites combinations, which is about one sixth of the species-sites (5472) used in our study. However, we can not agree with the highlighted conclusion that the significant decrease in $\mathrm{S}_{\mathrm{T}}$ using 15-year window is not sustained when examining longer term phenological responses to climate warming. On the contrary, we argue that the long-term linear trends may mask short-term phenological shifts.

According to the IPCC AR5, the period since the 1980s was very likely the warmest 30-year period of the last 800 years in the Northern Hemisphere (IPCC 2013); we therefore investigated the phenological changes during this warmest period ( $\mathrm{Fu}$ et al 2015). We welcome Wang et al's study that extended our analyses back to the 1950s, and found that $\mathrm{S}_{\mathrm{T}}$ slightly increased before the 1980s. However, they provided no explanation for this increase and did not quantify the intensity of chilling during this extended period. In addition, it should be noted that previous analyses of phenological data since 1753 in Switzerland also suggested long-term changes in $\mathrm{S}_{\mathrm{T}}$ based on 30year windows (Rutishauser et al 2008). This result actually supports our conclusion that changes in the number of chilling days can elicit changes in $\mathrm{S}_{\mathrm{T}}$. As shown in Fig. 1, the number of chilling days tended to increase before the 1980s, accompanying the increase in $\mathrm{S}_{\mathrm{T}}$, but both decreased simultaneously after the $1980 \mathrm{~s}$.

Wang and coauthors argue that the 15-year window used by Fu et al. may be too short to calculate $\mathrm{S}_{\mathrm{T}}$. This is not correct. The correlation coefficients between preseason temperature and the dates of leaf unfolding were generally statistically significant using 15 -year windows, i.e. a negative correlation was found at almost all sites, and the frequency of statistically significant negative correlations declined from $80 \%$ to $62.7 \%$ over the periods 1980-1994 and 1999-2013, respectively. Considering the fact that the $\mathrm{S}_{\mathrm{T}}$ should be statistically not different from zero at a site where the correlation is insignificant, the reduction in the fraction of significant correlations between these two periods suggests an increase in the number of sites at which the $\mathrm{S}_{\mathrm{T}}$ was statistically insignificant, indicating weakening $\mathrm{S}_{\mathrm{T}}$ at these sites over the period 1999-2013. Furthermore, we still found a significant reduction in $\mathrm{S}_{\mathrm{T}}$ even when only using the sites for which the leaf unfolding dates were statistically significantly correlated with preseason temperature (62.7\% of all sites) in both time periods (Fig. 2). This indicated that 15 years sufficed to detect changes of $\mathrm{S}_{\mathrm{T}}$.

Using longer-term time series can finally become a disadvantage, because shorter term, non-linear variation could become masked by it (Iler et al 2013). We noted that the response of leaf unfolding to temperature is non-linear, as Wang et al. confirmed. In this case, under conditions of climatic warming and over the considered time period (1951-2013), $\mathrm{S}_{\mathrm{T}}$ cannot be correctly estimated linear regressions covering longer time scales, such as the 30-year windows used by Wang et al. In other words, the assumption used in Wang et al. that one $\mathrm{S}_{\mathrm{T}}$ value can be representative for the entire 30-year period, 
is misleading, in particular when significant differences between the first and last 15 years are clearly present (Fig.3). Therefore, the analyses by Wang et al. do not invalidate our choice of a 15-year time window, neither our proposed mechanism linking this change in $\mathrm{S}_{\mathrm{T}}$ to reduced chilling days, nor the perspectives that the advancement of spring leaf unfolding will likely slow down in the future since the $\mathrm{S}_{\mathrm{T}}$ will be further reduced due to climate warming.

In conclusion, the additional analyses performed here with an enlarged data set only strengthen our finding that the $\mathrm{S}_{\mathrm{T}}$ of leaf unfolding is declining. We found that both the number of sites for which the leaf unfolding dates were statistically significantly related to the preseason temperature and the $\mathrm{S}_{\mathrm{T}}$ based on these sites support our finding that the $\mathrm{S}_{\mathrm{T}}$ significantly decreased over the past three decades. Furthermore, we argue that a 15-year time window is clearly more appropriate than a 30 -year time window to analyze the non-linear changes in the temperature sensitivity of leaf phenology.

\section{Reference}

Fu YH, Zhao H, Piao S, Peaucelle M, Peng S, Zhou G, et al. (2015)

Declining global warming effects on the phenology of spring leaf unfolding. Nature 526:104-107

Iler, A. M., Høye, T. T., Schmidt, N. M. \& Inouye, D. W. (2013) Long-term trends mask variation in the direction and magnitude of short-term phenological shifts. Am.

J. Bot 100, 1398-1406.

IPCC. Climate Change 2013: The Physical Science Basis. Contribution of Working Group I to the Fifth Assessment Report of the Intergovernmental Panel on Climate Change. (Cambridge University Press, 2013).

Rutishauser T, Luterbacher J, Defila C, Frank D,Wanner H (2008) Swiss spring plant phenology 2007: extremes, a multi-century perspective, and changes in temperature sensitivity. Geophys Res Lett 35:L5703

Wang H, Rutishauser T, Tao Z, Zhong S, Ge, Q, Dai J (2016) Impacts of global warming on phenology of spring leaf unfolding remain stable in the long run. 
Fig 1. Changes in chilling accumulation over the period 1951-2013. Following the method explained in $\mathrm{Fu}$ et al (2016), chilling accumulation was calculated as the number of days when the daily temperature was between 0 and $5{ }^{\circ} \mathrm{C}$ from 1 November to the mean date of leaf unfolding (MSOS). The dotted line indicates the changes in chilling accumulation over the period 1951-1979, and the dashed line indicates the changes over the period 1980-2013.

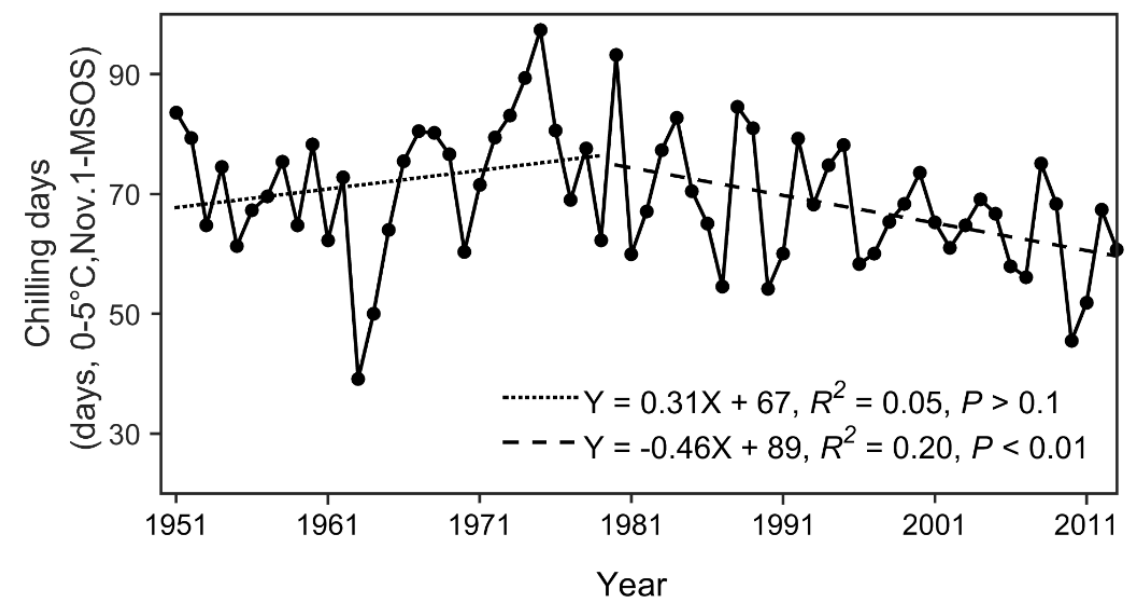


Fig 2. Temporal change of $S_{T}$ for individual species and for combined totals for all species across all sites with a 15-year moving window from 1980 to 2013. The black line indicates the average across all species, and the grey area indicates one s.d. either side of the mean. The dotted line indicates the linear regression. The $\mathrm{S}_{\mathrm{T}}$ was estimated using the sites for which the leaf unfolding dates were statistically significantly correlated with preseason temperature in both 1980-1994 and 1999-2013 periods.

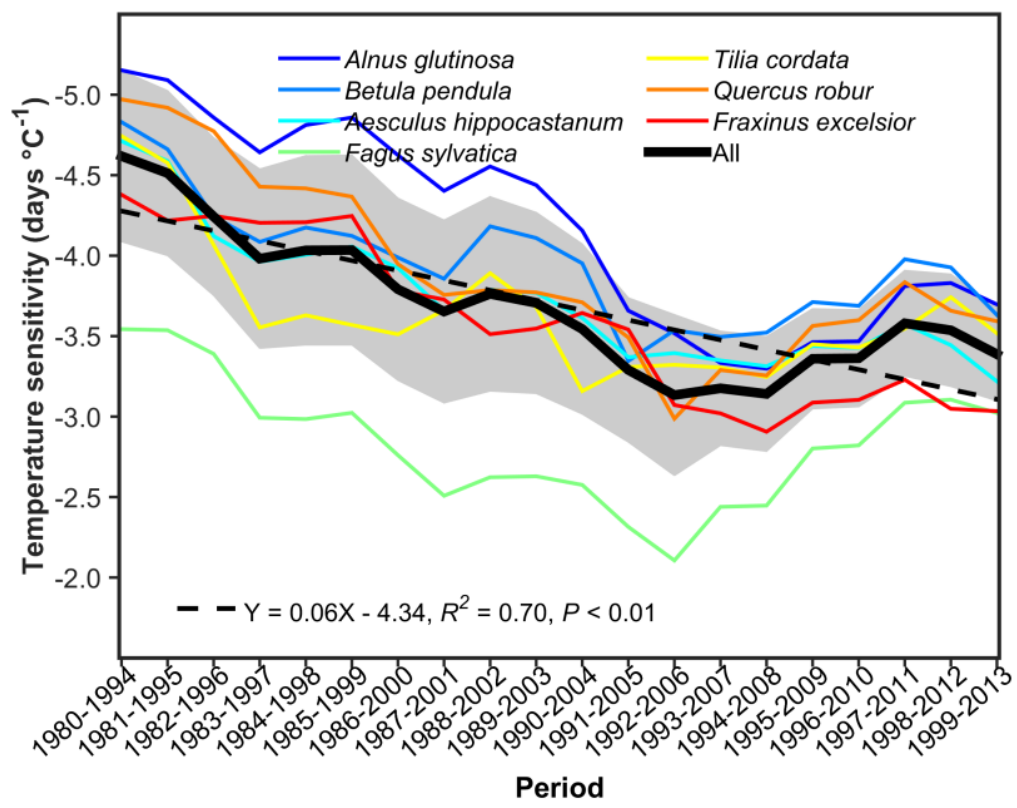


Fig. 3. Conceptual scheme of the nonlinear relationship between spring temperature and date of leaf unfolding. The black lines show the example of temperature sensitivity of leaf unfolding in two study periods in Fu et al (2016), i.e. 1980-1994 and 1999-2013. The red line shows the $\mathrm{S}_{\mathrm{T}}$ in Wang et al (2016) over the period 1980-2013.

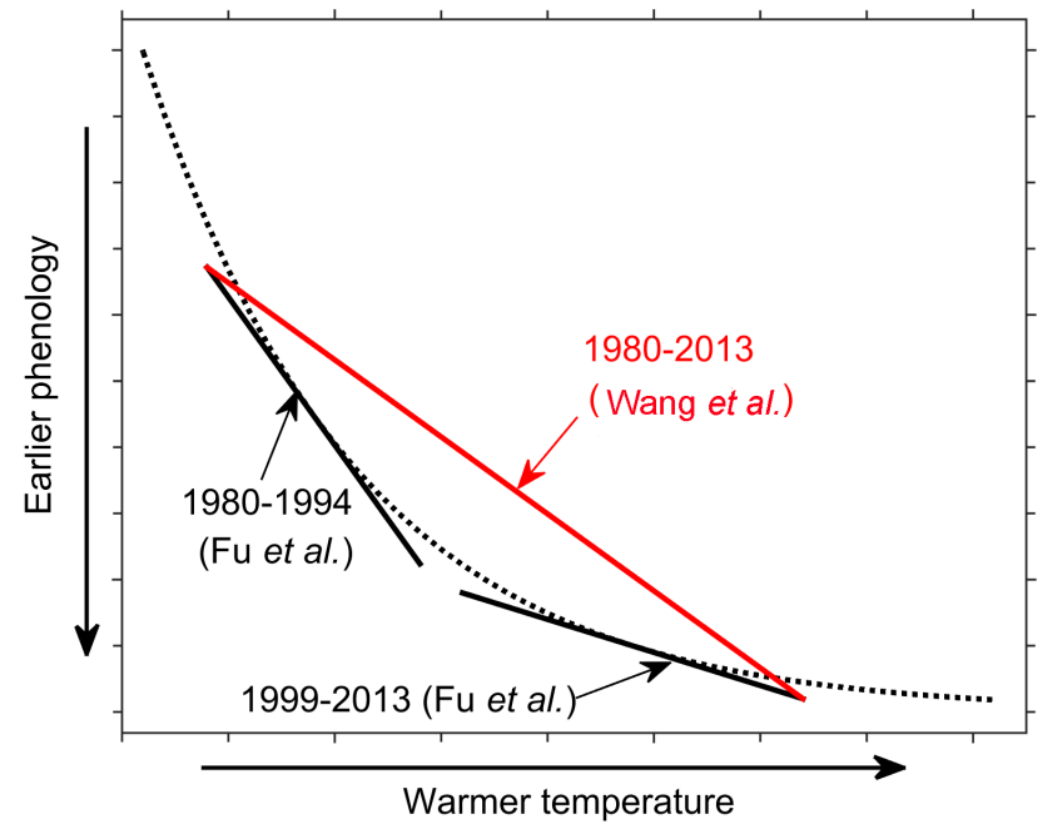

